\title{
Über die polarimetrische Bestimmung der Zuckerarten im Honig.
}

\author{
Erwiderung auf die Arbeit von P. Lehmann und H Stadlinger.
}

Von

Dr. J. Fiehe-Straßburg.

Auf die Arbeit von P. Lehmann und H. Stadlinger in dieser Zeitschrift ${ }^{1}$ ) erlaube ich mir folgendes zu erwidern: Der unbefangene Leser hat bei Durchsicht dieser Arbeit sicher das Gefühl, daß die Gründe, welche uns veranlaßten, die angeführten Honige zu beanstanden, nicht ausreichend gewesen seien und aus diesem Grunde keine Verurteilung des Angeklagten erfolgen konnte. Die Honige sind leider nur dem Laboratorium von Dr. Haenle zur Untersuchung übergeben worden und standen den übrigen Sachverständigen nur dis schriftlichen Analysenergebnisse zur Verfügung. Würden die Honige auch von anderer Seite untersucht worden sein, so hätten wir sicher nicht mit unserer Ansicht allein gestanden, denn solche offenbare Fälschungen wie der Honig $A_{4}$ hätte auch Stadlinger als Konfitüre und nicht als Honig bezeichnen müssen. Es handelte sich bei diesem Produkte nicht um eine Fälschung mit $16 \%$ Saccharose, wie Stadlinger meint, sondern um eine hochprozentige Fälschung mit Handelsinvertzucker, welche sofort am Geschmack kenntlich war. Wie sich im Verlaufe des Prozesses herausstellte, hatte der Angeklagte auch große Mengen Handelsinvertzucker ${ }^{2}$ ) bezogen. Zwei von mir untersuchte Invertzuckersirupe ${ }^{3}$ ) besaßen einen Saccharose-Gehalt von 29,1 und 32,3\%. Auf diesbezügliche Anfragen erfuhr ich, daß die Inversion bei den meisten Sirupen nicht weiter durchgeführt wird, um eine Krystallisation zu vermeiden. Der Honig $\mathbf{A}_{4}$ würde also nach der Polarisation der Lösung 1 - 2 im Soleil-Dubosc $q$-Apparat von $+7^{0}$ einem Gemisch von annähernd gleichen Teilen Fruchtzuckersyrup und links drehendem Blütenhonig entsprechen. Beweisend für unser Gutachten war das Verhalten des Honigs selbst; trotz vielmonatlichen Stehens war keine Spur von Krystallisation eingetreten. Würde die Rechtsdrehung von einem vorwiegenden Gehalt an Glykose herrühren, was nach der sachgemäßen Honigentnahme jedoch ganz ausgeschlossen war $^{4}$ ), so wäre zweifellos starke Krystallisation eingetreten, so aber hatte der Saccharosegehalt die Krystallisation verhindert. Die approximative Berechnung des Saccharose-Gehaltes was für uns nicht maßgebend (die Ziahl hat nicht einmal in unserem Gutachten Aufnahme gefunden), sondern in erster Linie Geschmack, Aroma und Konsistenz, verbunden mit der Rechtpolarisation und der hellen Farbe des Honigs, wodurch das Produkt zur Konfitüre gestempelt wurde. Bei der heutigen Art der Fälschungen mit Kunsthonig und Invertzuckersirup ist es unbedingt erforderlich auf die äußeren Eigenschaften eines Honigs ein größeres Gewicht zu legen, sonst sind den gewöhnlichsten Fälschungen Tür und Tor geöffnet. Betrachten wir z. B. die Zusammensetzung der Kunsthonige des Handels, so sehen wir, daß auf Grund chemischer Analyse in den meisten Fällen keine Beanstandung erfolgen kann:

1) Diese Zeitschrift 1907, 13, 397.

$\left.{ }^{2}\right)$ Nach Angabe zur Herstellung pharmazeutischer Präparate.

3) Aus Zürich und aus Straßburg.

4) Es war keine Trennung zwischen Glykose und Fruktose eingetreten. 


\begin{tabular}{|c|c|c|c|c|c|c|c|c|}
\hline $\begin{array}{c}\text { Kunsthonig, } \\
\text { bezogen von der Fabrik von }\end{array}$ & Wasser & $\begin{array}{l}\text { Saccha- } \\
\text { rose }\end{array}$ & $\begin{array}{l}\text { Invert- } \\
\text { zucker }\end{array}$ & $\begin{array}{l}\text { Nicht- } \\
\text { zueker }\end{array}$ & $\begin{array}{l}\text { Dex- } \\
\text { trin }\end{array}$ & $\begin{array}{c}\text { Gesamt- } \\
\text { säure als } \\
\text { A meisen- } \\
\text { säure }\end{array}$ & $\begin{array}{l}\text { Mine- } \\
\text { ral- } \\
\text { stoffe }\end{array}$ & $\begin{array}{c}\text { Polarisation } \\
\text { der Lösung } \\
1+2 \text { im } \\
\text { Soleil- } \\
\text { Daboseq } \\
\text { Apparat }\end{array}$ \\
\hline hrl & 9,98 & 5,32 & 73,00 & 1,70 & - & 0,059 & 0,0747 & -300 \\
\hline Winkelmann in Visselhövede ${ }^{1}$ ) & 21,60 & 3,80 & 70,02 & 4,58 & - & 0,150 & 0,0809 & -520 \\
\hline Paskal in Straßburg. . . . & 20,77 & 4,89 & 72,70 & 1,64 & 一 & 0,078 & 0,2050 & -500 \\
\hline Glorius in Leipzig & 24,60 & 14,10 & 60,75 & 0,55 & 0 & 0,055 & 0,4010 & +40 \\
\hline
\end{tabular}

Anders aber steht es, wenn der Chemiker Geschmack, Aroma, Farbe und Konsistenz eines Honigs mit in Betracht zieht. Hier sind wertvolle Kennzeichen vorhanden, welche unter Umständen ausschlaggebend sein müssen. Die Honiganalyse ist in mancher Beziehung mit der Weinanalyse zu vergleichen. Einen Honig und einen Wein analysenfest zu machen, ist nicht sebr schwer, dafür sorgen schon die Chemiker der Gegenseite. Die Geschmackprobe wird aber beim Wein für so wertvoll gehalten, daß der Richter auf Grund derselben zu einer Verurteilung kommt, auch wenn die Analysenzahlen keine weiteren Anbaltspunkte bieten. Erst kürzlich wurde hier im Elsass ein Weinhändler verurteilt, weil die Geschmacksprobe künstliche Parfümierung ergab. Bei allen Weinprozessen spielt die von einem Praktiker ausgeübte Geschmacksprobe die Hauptrolle. Warum soll nun bei der Honiganalyse die Geschmacksprobe, welche enorm wichtig ist, so ganz vernachlässigt werden? Etwa weil die meisten Chemiker keine Erfahrung darin haben? Dann soll man eben Praktiker zu Hilfe nehmen! Haenle, der sich seit 25 Jahren fast ausschließlich mit der Honiganalyse beschäftigt, hat immer großes Gewicht auf diese Geschmacksprobe gelegt. Das bedeutende Material, welches ihm von Imkern Deutschlands und auch vom Auslande zur Verfügung gestellt wurde, die eigene Versuchsstation von 5 Stöcken, gaben ihm Gelegenheit, sich nach dieser Seite hin reichlich Erfahrungen zu sammeln. Auf Grund dieser Erfahrungen werden im hiesigen Laboratorium Honige beurteilt, nicht aber allein auf einzelne Zahlen oder auf eine Erkennungsreaktion hin. Die Fälschung der Honige mit Invertzucker und Kunsthonigen hat zurzeit die größte Bedeutung. Stärkesirup und Saccharoseverfälschungen treten immer mehr in den Hintergrund. Ein mit Kunsthonig oder Handelsinvertzucker gefälschter Honig wird von uns in erster Linie am Geschmack und Aussehen erkannt. Confitürenaroma, Caramelgeschmack, die meist tiefgelbe Farbe der Kunsthonige (aus gegelbtem ${ }^{2}$ ) Zucker hergestellt) bieten uns wichtige Anhaltspunkte. Mit Invertzucker gefälschte Honige hinterlassen auf der Zunge einen intensiv süßen Geschmack (ähnlich wie Saccharin) während Naturhonige diese Eigenschaft nicht besitzen. Wird ein Honig mit Handelsinvertzucker verfälscht, so liegt die Drehung im Soleil-Duboscq-Apparate gewöhnlich einige Grad über oder unter 0, ähnlich wie bei Fütterungsprodukten. Bemerken muß jch aber, daß es auch Invertzuckersirupe im Handel gibt, welche stark links drehen; solche Sirupe sind aber seltener und werden nur auf ausdrückliche Bestellung

1) Dieser Honig ist einer der 8 verschiedenen Handelssorten dieser Firma.

2) Die Kunsthonige werden sehr häufig mit Azofarbstoffen gefärbt; man erkennt diese Farbstoffe an der Rotfärbung der wässerigen Lösung beim Zusatz von Salzsäure oder verdünnter Schwefelsäure. 
angefertigt. Unsere Erkennungsreaktion ${ }^{1}$ ) tritt erst in zweiter Linie in Kraft, bestätigt sie, was die Geschmacksprobe schon ergeben, so beanstanden wir den Honig.

$\mathrm{Da}$ ein solches Verfahren zur Beurteilung von Honigen, vom wissenschaftlichen Standpunkt aus betrachtet, nicht einwandfrei ist, soll nicht bestritten werden, solange wir aber kein besseres Verfahren kennen, muf einem solchen Untersuchungsverfahren schon eine gewisse Berechtigung zuerkannt werden.

Über die von Haenle aufgestellten Formeln zur Berechnung von Stärkesirup und Saccharose, welche Stadlinger als völlig unbrauchbar verwirft, habe ich folgendes zu bemerken: Die Formeln sind zweifellos in der auf Grund mathematischer Erwägungen von Stadlinger und Lehmann angegebenen Form richtiger; es ist aber von einer völlig genauen Bestimmung der Saccharose bezw. des Stärkesirups mit Hilfe dieser Formeln niemals die Rede gewesen, sondern stets von einer annähernden Bestimmung der Handelsverfälschungen. Haenle will grobe, zu Betrugszwecken ausgefübrte Fälschungen, welche wirklich in der Praxis vorkommen, erkennen und mit Hilfe seiner Formeln annähernd berechnen. Aus diesem Grunde ist die Berechnung von Stärkesirup und Saccharose bei Honigen, welche noch links drehen nie vorgenommen worden. Haenle sagt Seite 62 , Zeile 16 seines Buches ${ }^{2}$ ): Dreht ein heller, farbloser, gelber, gelbbrauner, grünlichgelber Honig das polarisierte Licht nach links, so ist derselbe mit Glykose oder Rüben-Zuckersirup nicht gefälscht. Auf Seite 54 erklärt er den Begriff Handelsverfälschungen, auf welche sich seine weitere Untersuchungsmethode aufbaut. Warum nehmen Lehmann und Stadlinger hierauf gar keine Rücksicht, wie man es doch bei einer objektiven Kritik verlangen muß? In den Beispielen, welche Lehmann und Stadlinger anführen, um die Unrichtigkeit der Haenle'schen Formel zu beweisen, ist immer wieder ein Honig zugrunde gelegt, welcher $-7^{0}$ Soleil-D uboseq dreht und welcher von Haenle als echt bezeichnet worden ist. Haenle hat dieses Produkt damals als echt bezeichnet, weil er es als echt von einem Imker erbalten hatte und er vor 12 Jahren auf ein Zuckerfütterungsprodukt, welches hier wabrscheinlich vorlag, noch kein so großes Gewicht legte. Solche Honige wurden aber schon damals von Haenle als seltene Ausnahmen unberücksichtigt gelassen uud hätten auch Lehmann und Stadlinger von der immerwährenden Anführung dieses Honigs besser Abstand genommen, besonders da von ihnen ein solches Zuckerfütterungsprodukt mit einer Drehung von $-6,51^{0}$ SoleilDuboscq in ihrer Tabelle Seite 416 unter No. 19 selbst angeführt wird. Was nun die Honige mit abnorm hoher Linksdrehung (über $-50^{\circ}$ Soleil-Duboscq) betrifft, so ist der Prozentsatz dieser Honige nach meinen Erfahrungen sehr gering.

Im Jahre 1906 wurden im hiesigen Laboratorium 317 Honige untersucht. Von diesen waren:
1. Echte Honige, von Imkern Deutschlands zur Honigsammlung geschickt . . .
2. Analysen eingesandter Honige

$\begin{array}{ccc}\text { Anzahl } & \overbrace{\text { der Proben }}^{\text {Polarisation }}{ }^{3}) \\ 101 & 0 & 1 \\ 216 & 0 & 11\end{array}$

Tannenhonige sind hier natürlich aufer Betracht gelassen.

1) Die Ley'sche Silberprobe liefert anerkanntermaßen günstige Ergebnisse (vergl. Zeitschrift angew. Chemie 1907, 20, 993); über unsere abgeänderte silberprobe werde ich noch später berichten.

$\left.{ }^{2}\right)$ O. Haenle, Chemie des Honigs. Strakburg 1896.

3) Die Polarisation ist stets nach Soleil-Duboscq angegeben. N. 07 . 
Von den 11 Honigen, welche mehr als $-50^{\circ}$ drehten, waren nur 4 deutscher Herkunft. Von den übrigen 7 waren 2 Kunsthonige, 3 verdorbene und saure Havannahonige und 2 Bretagnerhonige, welche dünnflüssig, braun, schwarz und so stark im Geschmack waren, daß sie völlig ungenießbar waren. Von den 4 deutschen Honigen waren 2 charakteristische Heidehonige und 2 gute Lindenblütenhonige. Der garantiert echte Honig, welcher in der obigen Tabelle unter No. 1 angegeben ist, war ein Heidehonig.

Es kommen für uns also in Wirklichkeit nur 5 Honige mit einer Polarisation über $-50^{\circ}$ von den 317 in Betracht $=1,6 \%$. Ich halte es deshalb auf Grund meiner Erfahrung nicht für richtig, den Honigen, welche über - $50^{0}$ Soleil-Dubosc $q$ drehen, eine solche Bedeutung beizulegen; es sind und bleiben Ausnahmen.

Wie groß sind nun die Differenzen, welche bei Berechnung nach der Ha en leschen Stärkesirupformel sich ergeben können?

Beispiel: Ein heller Honig gibt starke Dextrinreaktion und dreht in $331 / 3 \%$.iger Lösung im Soleil-Duboscq-Apparat +148 . Der Honig ist dadurch als eine Fälschung, bestehend aus Blütenhonig und Stärkesirup charakterisiert. Der Grad der Verfälschung wird nun nach $\mathrm{Haenle}$ durch die Formel $\frac{(\mathrm{P}+30) .3}{10}$ berechnet, in der $\mathrm{P}=$ Polarisation in Graden, $30=$ Normaldrehung der Blütenhonige ist.

Setzen wir nun statt der von Haenle angenommenen Normaldrehung von $30^{\circ}$ die Drehungen ein, die der ursprüngliche Honig möglicherweise besitzen könnte, so ergeben sich folgende Werte:

Angenommene Drehung der Lö-

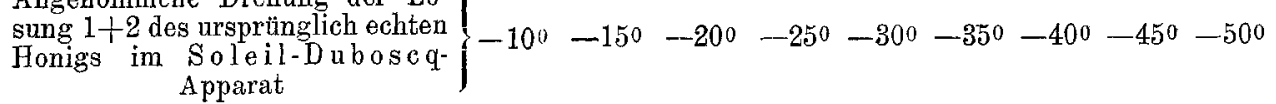

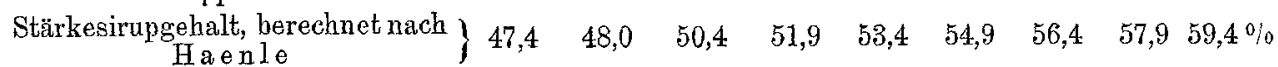

Das Gutachten nach Haenle würde lauten: „Der Honig ist mit etwa 50\% Stärkesirup verfälscht. Im Falle nun wirklich eine $47 \%$-ige oder eine $59 \%$-ige Fälschung rorläge, so würde dies bei einer Gerichtsverhandlung nicht viel ändern. Der Wert von $50 \%$ gibt aber dem Richter ein Bild von dem annähernden Grad der Verfälschung. Berücksichtigen wir ferner, daßs bei Verfälschungen mit Stärkesirup in Gutachten auf den Prozentsatz fast nie Rücksicht genommen wird - die vorliegenden Gutachten aus München und Kulmbach sprechen von einer starken Verfälschung mit Stärkesirup -, so wird man doch einer solchen annühernden Bestimmung ihre Existenzberechtigung nicht versagen können.

Das Haenle' sche Verfahren hat den Vorzug, in wenigen Minuten ausgeführt und beendet zu sein und in 80 von 100 Fällen Ergebnisse zu liefern, die vom wirklichen Verfälschungsgrad nur um wenige Prozente abweichen. Da sämtliche Bestimmungen von Stärkesirup im Honig nur annähernde Werte geben, kann man auch ein solches Verfahren anwenden, das den Vorzug der Einfachheit besitzt.

Im Beispiel 1 auf Seite 409 sagen. Stadlinger und Lehmann:

Dreht ein Honig mit schwacher Dextrinreaktion $-7^{0}$, so kann er sein:

1. entweder echt (ein Gemisch von Tannen- und Blütenhonig),

2. oder verfälscht und zwar:

a) mit $6,9 \%$ Glykose bei Zugrundelegung einer Drebung von $-30^{\circ}$, b) $" 14,4 \% " \quad " \quad " \quad " \quad,-55^{0}$.

Diese Angaben sind nicht zutreffend. Ein Gemisch von Tannen- und Blütenhonig wird von Haenle unter allen Umständen am Geschmack erkannt. Die eigen- 
artige Farbe des Tannenhonigs, die zähe Konsistenz, das Coniferenaroma sind eigenartige und so charakterische Merkmale, daß sie für den erfahrenen Honigchemiker nicht zu übersehen sind. Zu Punkt 2 ist zu bemerken, daß $\mathrm{H}$ aenle, wie er ausdrücklich Seite 62 seines Buches angibt, bei linksdrehenden Honigen (in diesem Falle also!) keine Glykosefälschung annimmt und berechnet ${ }^{1}$ ).

Im Beispiel 2 auf Seite 409 gehen Lehmann und Stadlinger von den gleichen Gesichtspunkten aus. Sie wollen, ganz gleichgültig, ob ein mit Glykose gefälschter Blütenhonig, oder ein charakteristischer Tannenhonig vorliegt, nur nach der Drehung, nicht aber nach Farbe, Aroma etc. urteilen. Eine solche Anwendung der Haenleschen Methode ist natürlich völlig verfehlt und entspricht keineswegs dem Sinne, in welchem sie gehandhabt werden soll.

In ähnlicher Weise gehen Lehmann nnd Stadlinger bei Berechnung des Saccharosegehaltes nach der Haenle'schen Formel vor. Im Beispiel 1 auf Seite 410 legen sie einen anormalen Honig, welcher $-7^{0}$ Soleil-Duboscq dreht, zugrunde und berechnen bei diesem Honig den Saccharosegehalt mit Hilfe der Formel, obschon, wie Haenle angibt, die Anwendung der Formel erst bei Rechtsdrehung eintritt. Aber augenommen, ich berechne den Saccharosegehalt und erhalte 10,3\% Saccharose, warum soll dieses so völlig unrichtig sein? Lehmann und Stadlinger geben in ibrer Tabelle Seite 416 und 417 unter No. 19 selbst einen Honig an, der fast genau dieselbe Drehung, nämlich $-6,51^{0}$, besitzt. Berechnen wir hier den Saccharosegehalt nach Haenle, so erhalten wir 10,56\% Saccharose; aus der Tabelle ist nun aber ersichtlich, daß in Wirklichkeit $10,32 \%$ vorhanden sind. Die beiden Ergebnisse stimmen also vorzüglich überein. Von einem Gemisch von Blüten- und Tannenhonig kann in diesem Falle überhaupt keine Rede sein, da dieses am Geschmack, am Aroma und am Dextringehalt erkannt und ausgeschaltet wird. Die Fälschung mit Hattenheimer Fruchtzucker will ich übergehen, da eine solche doch ganz verschieden ist von einer Fälschung mit Saccharose und mit der Formel gar nichts zu tun hat.

Im Beispiel 2 auf Seite 411 nehmen Lehmann und Stadlinger einen anormalen Honig an, welcher $-55^{0}$ dreht; sie fälschen denselben mit $15 \%$ Saccharosesirup $=9 \%$ Saccharose und berechnen dann bei diesem Produkt, welches $-26^{0}$ dreht, nach der Haenle'schen Formel die Saccharose. Sie verfahren also genau entgegengesetzt, wie $\mathrm{Haenle}$ angibt. Bei einer solchen Anwendung der Haenleschen Methode sind natürlich keine richtigen Ergebnisse möglich. Bei den gesamten Beispielen werden völlig anormale Honige zugrunde gelegt und Haenle's erste Bedingung Handelsfälschungen annähernd zu berechnen unberücksichtigt gelassen. So wird im Beispiel 3 auf Seite 412 auf die Unrichtigkeit der Haenleschen Formel bingewiesen, wenn ein ursprünglich echter Honig der Fälschung zugrunde liegt, der entweder $-7^{0}$, oder $-30^{0}$ oder $-55^{0}$ dreht. Ein Honig aber, der $-7^{0}$ dreht und keinen Tannenhonig enthält, kann doch nur seine, geringe Drehung von einem anormal hohen Saccharosegehalt her besitzen (sachgemäße Honigentnahme vorausgesetzt) und würde sich dementsprechend die Rechnung ganz anders gestalten; es würde sich der vorhandene Prozentsatz an Saccharose zur Fälschung hinzuaddieren und die Ergebnisse würden sich annähernd decken. Zum Schlusse ihrer Arbeit berechnen Lehmann und Stadlinger bei einem Honig, der — 29,10 Ruicksicht.

1) Haenle nimmt, wie wiederholt hervorgehoben sei, nur auf Handelsverfälschungen 
dreht, nach der Haenle'schen Formel die Saccharose. Auf diese unrichtige Anwendung des Verfahrens habe ich schon wiederholt hingewiesen. Auf die wirklicken Differenzen, welche sich bei Anwendung der Saccharoseformel ergeben, hat Haenle auf Seite 120 seines Buches selbst hingewiesen. Auch $\mathrm{Haenle}$ stimmt darin mit Lehmann und Stadlinger vollkommen überein, daß eine genaue polarimetrische Saccbarosebestimmung im Honig nur mit Hilfe des Inversionsverfahrens möglich sei. Wenn aber Lehmann und Stadlinger auf Grund ihrer Arbeit die Behauptung aufstellen, daß die Anwendung der $\mathrm{Ha}$ en le' schen Formeln zu völligen Trugschlüssen führen könne, so ist dieses ein Irrtum auf ihrer Seite und der Beweis für ihre Behauptung keineswegs erbracht.

Die Haenle'schen Formeln setzen allerdings voraus, daß der Chemiker Erfahrungen in der Honigchemie besitzt und imstande ist, einen Honig nach Aussehen, Geschmack und Aroma zu beurteilen und zu klassifizieren. Ohne diese Fähigkeit ist nach meiner Ansicht überhaupt keine richtige Honiganalyse möglich. Der Honig verlangt als Produkt des organischen Lebens eine individuelle Beurteilung je nach den Jahren und nach der Tracht.

\section{Beriehtigung.}

In der vierten, mit Herrn Prof. Dr. A. Bömer bearbeiteten Auflage meiner ,Chemie der menschlichen Nahrungs- und Genußmittel 1903. I. Band, S. 1032 ist eine Analyse von H a ferKakao der Kasseler Hafer-Kakao-Eabrik, Hausen \& Co. A.G. in Kassel mit einem Wassergehalt $\nabla 0 n$ 11,64\% aufgeführt. Obschon es sich um eine einzelne Probe handelt, die selbstverständlich nicht für den Durchschnitt eines Fabrikates maß́gebend sein kann, so wird diese Analyse doch wegen des bohen Wassergehaltes von der Konkurrenz in unlauterer Weise gegen die Ware der genannten Fabrik ausgenutzt. Letztere sendet mir nun acht Originalatteste der Landwirtschaftlichen Versuchsstation in Marburg uber ibren Haferkakao, welche die Proben in den Jahren 1904-1907 aus versehiedenen Geschäften Marburgs selbst entnommen und dafür im Mittel gefunden hat:

$\begin{array}{cccccc}\text { Wasser } & \text { Protein } & \text { Fett } & \text { Kohlenhydrate } & \text { Rohfaser } & \text { Asche } \\ 7,18 \% & 16,38 \% & 16,82 \% & 52,45 \% & 2,17 \% & 5,00 \%\end{array}$

Die Schwankungen im Wassergehalt haben in diesen Proben nur 6,13-8,96\% betragen.

Münster i. W. den 15. Juli 1907.

J. König.

\section{Referate.}

\section{Zucker, Zuckerwaren und künstliche Sïßstoffe.}

K. Andrlik und J. Urban: Belege für das Übergehen des schädlichen Stickstoffes aus der Rübe in die Säfte, für dessen Stabilität während der Saftreinigung und seine Zunahme bei längerer Lagerung der Rübe. (Zeitschr. Zuckerind. in Böhmen 1906, 30, 282; Österr.ungar. Zeitschr. Zuckerind. u. Landw. 1906, 35, 383-385.) — Der schädliche Stickstoff aus der Rübe geht fast vollkommen in den Preßsaft über und-kann auch während der Reinigung durch die Saturation nicht beseitigt werden. Bei der Diffusion gelangen nur etwa $90 \%$ von dem Stickstoff der Rübe in den Saft; an schädlichem Stickstoff reichere Rüben liefern Diffusionssäfte von schlechterer Beschaffenheit; auch der Gesamtstickstoff der Rübe beeinflußt manchmal die Qualität der Säfte. Bei längerer Lagerung 